Bull. Austral. Math. Soc.

VOL. 61 (2000) [263-266]

\title{
VERIFYING THE INDEPENDENCE OF PARTITIONS OF A PROBABILITY SPACE
}

\author{
S.B. Mulay and C.G. Wagner
}

Let $\left\{E_{1}, \ldots, E_{r}\right\}$ and $\left\{F_{1}, \ldots, F_{s}\right\}$ be partitions of a probability space. We exhibit a natural bijection from the set of efficient ways of verifying the independence of such partitions to the set of spanning trees of the complete bipartite graph $K_{r, s}$.

\section{INTRODUCTION}

In what follows, $(\Omega, \Sigma, p)$ is a probability space and $[n]:=\{1,2, \ldots, n\}$. Partitions $\left\{E_{i}: i \in[r]\right\}$ and $\left\{F_{j}: j \in[s]\right\}$ of $\Omega$, with $E_{i}, F_{j} \in \Sigma$, are said to be independent (with respect to $p$ ) if

$$
p\left(E_{i} \cap F_{j}\right)=p\left(E_{i}\right) p\left(F_{j}\right)
$$

for all $(i, j) \in[r] \times[s]$. Of course, one need not check all $r s$ instances of $(1)$ in order to verify independence. It is easy to see, for example, that if (1) holds for all $(i, j) \in[r-1] \times[s-1]$, then the partitions in question are independent. Let us call a subset $\mathcal{N}$ of $[r] \times[s]$ negligible when, if $(1)$ holds for all $(i, j) \in \mathcal{N}^{c}$, then it holds for all $(i, j) \in \mathcal{N}$ as well. We show in this note that there is a natural bijection from the family of maximal negligible subsets of $[r] \times[s]$ to the family of spanning trees of the complete bipartite graph $K_{r, s}$. It follows that there are $r^{s-1} s^{r-1}$ efficient ways to verify the independence of the aforementioned partitions.

\section{Negligibility and Linear INDEPENDENCE}

For all $i \in[r]$, let $X_{i}$ be the $(r+s)$-dimensional unit column vector with a one in the $i$ th position and zeros elsewhere, and for all $j \in[s]$, let $Y_{j}$ be the $(r+s)$ dimensional unit column vector with a one in the $(r+j)$ th position and zeros elsewhere. Then

$$
\sum_{(i, j) \in[r] \times[s]} p\left(E_{i} \cap F_{j}\right)\left(X_{i}+Y_{j}\right)=\sum_{i \in[r]} p\left(E_{i}\right) X_{i}+\sum_{j \in[s]} p\left(F_{j}\right) Y_{j}
$$

Received 31st May, 1999

Research supported by the National Science Foundation (SBR-9528893).

Copyright Clearance Centre, Inc. Serial-fee code: 0004-9727/00 \$A2.00+0.00. 
as a consequence of the familiar formulas for the marginal probabilities $p\left(E_{i}\right)$ and $p\left(F_{j}\right)$.

Suppose that $\mathcal{N} \subset[r] \times[s]$, and that $p\left(E_{i} \cap F_{j}\right)=p\left(E_{i}\right) p\left(F_{j}\right)$ for all $(i, j) \in \mathcal{N}^{c}$. Then (2) becomes

$$
\begin{aligned}
\sum_{(i, j) \in \mathcal{N}} p\left(E_{i} \cap F_{j}\right)\left(X_{i}+Y_{j}\right)=\sum_{i \in[r]} p\left(E_{i}\right) X_{i}+\sum_{j \in[s]} p\left(F_{j}\right) Y_{j} \\
-\sum_{(i, j) \in \mathcal{N} c} p\left(E_{i}\right) p\left(F_{j}\right)\left(X_{i}+Y_{j}\right)
\end{aligned}
$$

Regard the quantities $p\left(E_{i} \cap F_{j}\right)$, where $(i, j) \in \mathcal{N}$, as unknowns. It is clear that $p\left(E_{i} \cap F_{j}\right)=p\left(E_{i}\right) p\left(F_{j}\right)$ for all $(i, j) \in \mathcal{N}$ furnishes a solution of $(3) . \mathcal{N}$ is negligible if and only if this is the only solution of (3), and the latter condition clearly obtains if and and only if $\left\{X_{i}+Y_{j}:(i, j) \in \mathcal{N}\right\}$ is a linearly independent subset of $V$, the subspace of $\mathbb{R}^{r+s}$ spanned by $\left\{X_{i}+Y_{j}:(i, j) \in[r] \times[s]\right\}$. Consequently, $\mathcal{N}$ is a maximal negligible subset of $[r] \times[s]$ if and only if $\left\{X_{i}+Y_{j}:(i, j) \in \mathcal{N}\right\}$ is a basis of $V$.

It is easy to see that the dimension of $V$ is $r+s-1$. In particular, the set of column vectors $\left\{X_{1}+Y_{s}, X_{2}+Y_{s}, \ldots, X_{r-1}+Y_{s}, X_{r}+Y_{1}, X_{r}+Y_{2}, \ldots, X_{r}+Y_{s}\right\}$ is a basis of $V$. This set spans $V$ since $X_{i}+Y_{j}=\left(X_{i}+Y_{s}\right)+\left(X_{r}+Y_{j}\right)-\left(X_{r}+Y_{s}\right)$. It is linearly independent as a simple consequence of the linear independence of $\left\{X_{1}, \ldots, X_{r}, Y_{1}, \ldots, Y_{s}\right\}$. In the next section we present a graphical characterisation of bases of $V$ consisting of vectors of the form $X_{i}+Y_{j}$.

\section{A Natural Bijection}

We assume in this section familiarity with the basic terminology and elementary results of graph theory, as described, for example, in [3]. In particular, we use the fact that if all vertices of a graph have degree at least two, then the graph contains a cycle [3, Lemma 1.2.18], and the fact that a graph with $n$ vertices is a tree if and only if it is acyclic and has $n-1$ edges [3, Theorem 2.13].

Consider the complete bipartite graph $K_{r, s}$ with vertex set $\mathcal{V}=\left\{X_{1}, \ldots, X_{r}\right.$; $\left.Y_{1}, \ldots, Y_{s}\right\}$ and edge set $\mathcal{E}=\left\{\left\{X_{i}, Y_{j}\right\}:(i, j) \in[r] \times[s]\right\}$. To each $\mathcal{S} \subset[r] \times[s]$ we associate the subgraph of $K_{r, s}$ having vertex set $\mathcal{V}(\mathcal{S})=\bigcup_{(i, j) \in \mathcal{S}}\left\{X_{i}, Y_{j}\right\}$ and edge set $\mathcal{E}(\mathcal{S})=\left\{\left\{X_{i}, Y_{j}\right\} ;(i, j) \in \mathcal{S}\right\}$. The map $\mathcal{S} \mapsto(\mathcal{V}(\mathcal{S}), \mathcal{E}(\mathcal{S}))$ is clearly an injection from $2^{[r] \times[s]}$ into the set of all subgraphs of $K_{r, s}$.

LEMMA. The set of column vectors $S(\mathcal{S})=\left\{X_{i}+Y_{j}:(i, j) \in \mathcal{S}\right\}$ is linearly dependent if and only if $(\mathcal{V}(\mathcal{S}), \mathcal{E}(\mathcal{S}))$ contains a cycle.

ProOF: Sufficiency. Suppose, with no loss of generality, that $(\mathcal{V}(\mathcal{S}), \mathcal{E}(\mathcal{S}))$ contains the cycle $X_{i_{1}}, Y_{i_{1}}, X_{i_{2}}, Y_{i_{2}}, \ldots, X_{i_{n}}, Y_{i_{n}}, X_{i_{1}}$. Since then $X_{i_{1}}+Y_{i_{1}}, X_{i_{2}}+Y_{i_{1}}, X_{i_{2}}+$ 
$Y_{i_{2}}, \ldots, X_{i_{n}}+Y_{i_{n}}$ and $X_{i_{1}}+Y_{i_{n}} \in S(\mathcal{S})$ and $\left(X_{i_{1}}+Y_{i_{1}}\right)-\left(X_{i_{2}}+Y_{i_{1}}\right)+\left(X_{i_{2}}+Y_{i_{2}}\right)-$ $\cdots+\left(X_{i_{n}}+Y_{i_{n}}\right)-\left(X_{i_{1}}+Y_{i_{n}}\right)=0$, it follows that $S(S)$ is linearly dependent.

Necessity. Suppose that $S(\mathcal{S})$ is linearly dependent. Then there exists a nonempty subset $\mathcal{S}^{+}$of $\mathcal{S}$ and, for each $(i, j) \in \mathcal{S}^{+}$, a nonzero real number $\alpha_{i j}$ such that

$$
\sum_{(i, j) \in \mathcal{S}^{+}} \alpha_{i j}\left(X_{i}+Y_{j}\right)=0
$$

Consider the graph $\left(\mathcal{V}\left(\mathcal{S}^{+}\right), \mathcal{E}\left(\mathcal{S}^{+}\right)\right)$. Clearly, every vertex in $\mathcal{V}\left(\mathcal{S}^{+}\right)$has degree at least one. Suppose some vertex, say $X_{i^{*}}$, has degree one, belonging only to the edge $\left\{X_{i^{*}}, Y_{j^{*}}\right\}$. Then $X_{i}^{*}$ occurs just once in (4), with the nonzero coefficient $\alpha_{i^{*} j^{*}}$. This implies that $X_{i^{*}}$ is a linear combination of $\left\{X_{1}, \ldots, X_{r}, Y_{1}, \ldots, Y_{s}\right\} \backslash\left\{X_{i^{*}}\right\}$, contradicting the linear independence of $\left\{X_{1}, \ldots, X_{r}, Y_{1}, \ldots, Y_{s}\right\}$. Hence every vertex in $\mathcal{V}\left(\mathcal{S}^{+}\right)$ has degree at least two, and so $\left(\mathcal{V}\left(\mathcal{S}^{+}\right), \mathcal{E}\left(\mathcal{S}^{+}\right)\right)$, and thus $(\mathcal{V}(\mathcal{S}), \mathcal{E}(\mathcal{S}))$, contains a cycle.

Students of matroid theory will not be surprised by the above lemma. Indeed, it establishes a special case of a much more general result, namely the fact that the cycle matroid of every graph has a vectorial representation [2, Section 9.5]. We may now establish the main result of this note.

THEOREM. The map $\mathcal{N} \mapsto(\mathcal{V}(\mathcal{N}), \mathcal{E}(\mathcal{N}))$ is a bijection from the family of all maximal negligible subsets of $[r] \times[s]$ to the set of all spanning trees of $K_{r, s}$.

Proof: If $\mathcal{N}$ is a maximal negligible subset of $[r] \times[s]$, then, as shown in Section 2 above, $S(\mathcal{N})=\left\{X_{i}+Y_{j}:(i, j) \in \mathcal{N}\right\}$ is a basis of $V$, the $(r+s-1)$-dimensional subspace of $\mathbb{R}^{r+s}$ spanned by $\left\{X_{i}+Y_{j}:(i, j) \in[r] \times[s]\right\}$. By the preceding lemma, $(\mathcal{V}(\mathcal{N}), \mathcal{E}(\mathcal{N}))$ is acyclic. Clearly, $|\mathcal{E}(\mathcal{N})|=|S(\mathcal{N})|=r+s-1$. Also, $\mathcal{V}(\mathcal{N})=$ $\left\{X_{1}, \ldots, X_{r}, Y_{1}, \ldots, Y_{s}\right\}$, for if not, $S(\mathcal{N})$ would not span $V$. Hence, $|\mathcal{V}(\mathcal{N})|=r+s$. It follows that $(\mathcal{V}(\mathcal{N}), \mathcal{E}(\mathcal{N}))$ is a tree with the same vertex set as $K_{r, s}$, and edge set contained in the edge set of $K_{r, s}$, that is, a spanning tree of $K_{r, s}$.

As a restriction of the injective map $\mathcal{S} \mapsto(\mathcal{V}(\mathcal{S}), \mathcal{E}(\mathcal{S}))$, the map $\mathcal{N} \mapsto(\mathcal{V}(\mathcal{N}), \mathcal{E}(\mathcal{N}))$ is injective. It remains only to show that this map is surjective. Let $(\mathcal{V}, \mathcal{E})$ be a spanning tree of $K_{r, s}$, so that $\mathcal{V}=\left\{X_{1}, \ldots, X_{r}, Y_{1}, \ldots, Y_{s}\right\}$ and $\mathcal{E} \subset\left\{\left\{X_{i}, Y_{j}\right\}\right.$ : $(i, j) \in[r] \times[s]\}$. Then $|\mathcal{E}|=r+s-1$. Suppose that $\mathcal{E}=\left\{\left\{X_{i}, Y_{j}\right\}:(i, j) \in \mathcal{N}\right\}$ where $\mathcal{N} \subset[r] \times[s] . \quad$ Clearly, $\mathcal{V}(\mathcal{N})=\mathcal{V}$ and $\mathcal{E}(\mathcal{N})=\mathcal{E}$. By the lemma, $S(\mathcal{N})=\left\{X_{i}+Y_{j}:(i, j) \in \mathcal{N}\right\}$ is linearly independent since $(\mathcal{V}(\mathcal{N}), \mathcal{E}(\mathcal{N}))$ is acyclic. Since $|S(\mathcal{N})|=|\mathcal{N}|=|\mathcal{E}|=r+s-1, S(\mathcal{N})$ is a basis of $V$. Hence by the results of Section 2 above, $\mathcal{N}$ is a maximal negligible subset of $[r] \times[s]$, which completes the proof of surjectivity.

Since the complete bipartite graph $K_{r, s}$ has $r^{s-1} s^{r-1}$ spanning trees [1], it follows that there are $r^{s-1} s^{r-1}$ efficient ways to verify the independence of partitions 
$\left\{E_{1}, \ldots, E_{r}\right\}$ and $\left\{F_{1}, \ldots, F_{s}\right\}$.

REMARK 1. The foregoing analysis could have be carried out with $\left\{X_{1}, \ldots, X_{\tau}\right.$, $\left.Y_{1}, \ldots, Y_{s}\right\}$ being any set of distinct indeterminates over $\mathbb{R}$.

REMARK 2. Our characterisation in Section 2 above of the efficient ways of verifying the independence of two partitions of a probability space may be generalised to the case of three or more partitions. In the case of partitions $\left\{E_{1}, \ldots, E_{r}\right\},\left\{F_{1}, \ldots, F_{s}\right\}$, and $\left\{G_{1}, \ldots, G_{t}\right\}$, for example, maximal negligible subsets of $[r] \times[s] \times[t]$ correspond to bases of the vector space generated by $\left\{X_{i}+Y_{j}+Z_{k}:(i, j, k) \in[r] \times[s] \times[t]\right\}$ comprised of vectors of the form $X_{i}+Y_{j}+Z_{k}$. The problem of enumerating bases of this type has, as far as we know, not been solved. The vectors comprising such bases correspond in a natural way to edges of a hypergraph, but it is not clear what sorts of hypergraphs arise in this way, or whether they facilitate the enumeration in question.

\section{REFERENCES}

[1] H. Scoins, 'The number of trees with nodes of alternate parity', Proc. Cambridge Philos. Soc. 58 (1962), 12-16.

[2] D. Welsh, Matroid theory (Academic Press, London, 1976).

[3] D. West, Introduction to graph theory (Prentice Hall, Upper Saddle River, NJ, 1996).

Department of Mathematics The University of Tennessee Knoxville, TN 37996-1300

United States of America 